\title{
Shedding of the endothelial glycocalyx during cardiac surgery: On-pump versus off-pump coronary artery bypass graft surgery
}

\author{
Dirk Bruegger, MD, ${ }^{\mathrm{a}, *}$ Markus Rehm, MD, ${ }^{\mathrm{a}, *}$ Jan Abicht, MD, ${ }^{\mathrm{a}}$ Joern Oliver Paul, MD, ${ }^{\mathrm{a}}$ \\ Mechthild Stoeckelhuber, MD, PhD, ${ }^{\mathrm{b}}$ Markus Pfirrmann, MSc, ${ }^{\mathrm{c}}$ Bruno Reichart, MD, ${ }^{\mathrm{d}}$ \\ Bernhard F. Becker, MD, PhD, ${ }^{\mathrm{e}}$ and Frank Christ, MD, ${ }^{\mathrm{a}}$ Munich, Germany
}

\begin{abstract}
A healthy vascular endothelium is coated by the endothelial glycocalyx. Its main constituents are transmembrane syndecans and bound heparan sulfates. In vivo the glycocalyx binds plasma proteins, forming the endothelial surface layer, which has a thickness of 500 to $1000 \mathrm{~nm}$ in microvessels. ${ }^{1}$ This large structure maintains the physiologic permeability barrier and prevents leukocyte and platelet adhesion, thereby mitigating inflammation and tissue edema. ${ }^{1,2}$

Very recently, we provided the first evidence for an acute destruction of the endothelial glycocalyx in patients undergoing aortic surgery associated with ischemia reperfusion injury. ${ }^{3}$ Avoiding cardiopulmonary bypass (CPB) and cardioplegic arrest, including ischemia and reperfusion, should presumably reduce shedding of the endothelial glycocalyx. In the present study shedding of components of the endothelial glycocalyx (heparan sulfate and syndecan-1) was investigated in patients undergoing coronary artery bypass surgery with and without $\mathrm{CPB}$.
\end{abstract}

\section{CLINICAL SUMMARY}

Patients referred for elective coronary artery bypass surgery were prospectively randomized to on-pump conventional coronary artery bypass $(\mathrm{CCAB} ; \mathrm{n}=15)$ or off-pump coronary artery bypass $(\mathrm{OPCAB} ; \mathrm{n}=15)$ surgery. Exclusion criteria were former or combined cardiac procedures and renal or hepatic dysfunction. Before surgical intervention, written informed consent was obtained from each patient. The study was approved by the ethics committee of our institution. $\mathrm{CCAB}$ and $\mathrm{OPCAB}$ operations were performed through a median sternotomy. Mild hypothermia $\left(32.0^{\circ} \mathrm{C}\right)$ was used

\footnotetext{
From the Clinic of Anesthesiology $y^{a}$; the Department of Anatomy ${ }^{b}$; the Department of Medical Informatics, Biometry, and Epidemiology ${ }^{\mathrm{c}}$; the Department of Cardiac Surgery $^{\mathrm{d}}$; and the Institute of Physiology, ${ }^{\mathrm{e}}$ Ludwig-Maximilians-University, Munich, Germany.

This work was performed with departmental research funding provided by the Government of Bavaria (Bavarian State Ministry of Science, Research, and the Arts, Munich, Germany) and a grant provided by the Friedrich-Baur-Stiftung, Munich, Germany.

*D.B. and M.R. contributed equally to this article.

Received for publication July 8, 2008; revisions received July 22, 2008; available ahead of print Feb 5, 2009.

Address for reprints: Dirk Bruegger, MD, Clinic of Anesthesiology, Ludwig-Maximilians-University, Marchioninistr. 15, D-81377 Munich, Germany (E-mail: dirk. bruegger@med.uni-muenchen.de).

J Thorac Cardiovasc Surg 2009;138:1445-7

0022-5223/\$36.00

Copyright $\Subset 2009$ by The American Association for Thoracic Surgery

doi:10.1016/j.jtcvs.2008.07.063
}

for $\mathrm{CCAB}$ surgery, whereas temperature was maintained at greater than $35.5^{\circ} \mathrm{C}$ for $\mathrm{OPCAB}$ surgery. In patients undergoing $\mathrm{CCAB}$ surgery, blood samples were taken before (T0) and after (T1) induction of anesthesia and before surgical incision, at the onset of CPB (T2), after removal of the aortic crossclamp during completion of proximal anastomoses (T3), 5 minutes after CPB and at the beginning of surgical hemostasis (T4), and at skin closure (T5). The corresponding time points for patients undergoing OPCAB surgery were before (T0) and after (T1) induction of anesthesia and before surgical incision, after the first application of the stabilizer (T2), during completion of the proximal anastomoses with the heart returned to its natural position (T3), at the start of surgical hemostasis (T4), and at skin closure (T5). Plasma blood samples were separated by means of centrifugation and stored at $-80^{\circ} \mathrm{C}$ until assay. Syndecan- 1 and heparan sulfate concentrations were determined by means of ELISA. ${ }^{3}$

Saphenous veins left over after coronary artery bypass grafting were obtained after the last proximal anastomosis was performed and stored in $4 \%$ formaldehyde solution for 24 hours.

There were no differences regarding patients' demographic data, duration of anesthesia, and operation. The clinical course of all patients was uneventful. Figure 1 shows the measured syndecan- 1 and heparan sulfate concentrations in the plasma of both groups. Anesthesia and initiation of surgical intervention caused no significant changes. In CCAB surgery the removal of the aortic crossclamp during reperfusion of the heart and lungs led to significant median 2- and 4-fold increases in syndecan-1 and heparan sulfate concentrations, respectively. There were further increases 5 minutes after termination of CPB. Surprisingly, in patients undergoing OPCAB surgery, there were also significant increases in median syndecan-1 (4-fold) and heparan sulfate (2-fold) concentrations during completion of the proximal anastomoses. Syndecan-1 and heparan sulfate concentrations had returned to basal levels by the end of the operation in both the CCAB and OPCAB groups.

Light microscopic photographs showing the endothelial glycocalyx of a human saphenous vein and its adventitial vessels are depicted in Figure 2. Heparan sulfate and syndecan-1 were detected at the luminal side of the endothelium.

\section{DISCUSSION}

The major finding of this study is that components of the endothelial glycocalyx, syndecan-1 and heparan sulfate, are released from the tissue and can be detected in the blood of 

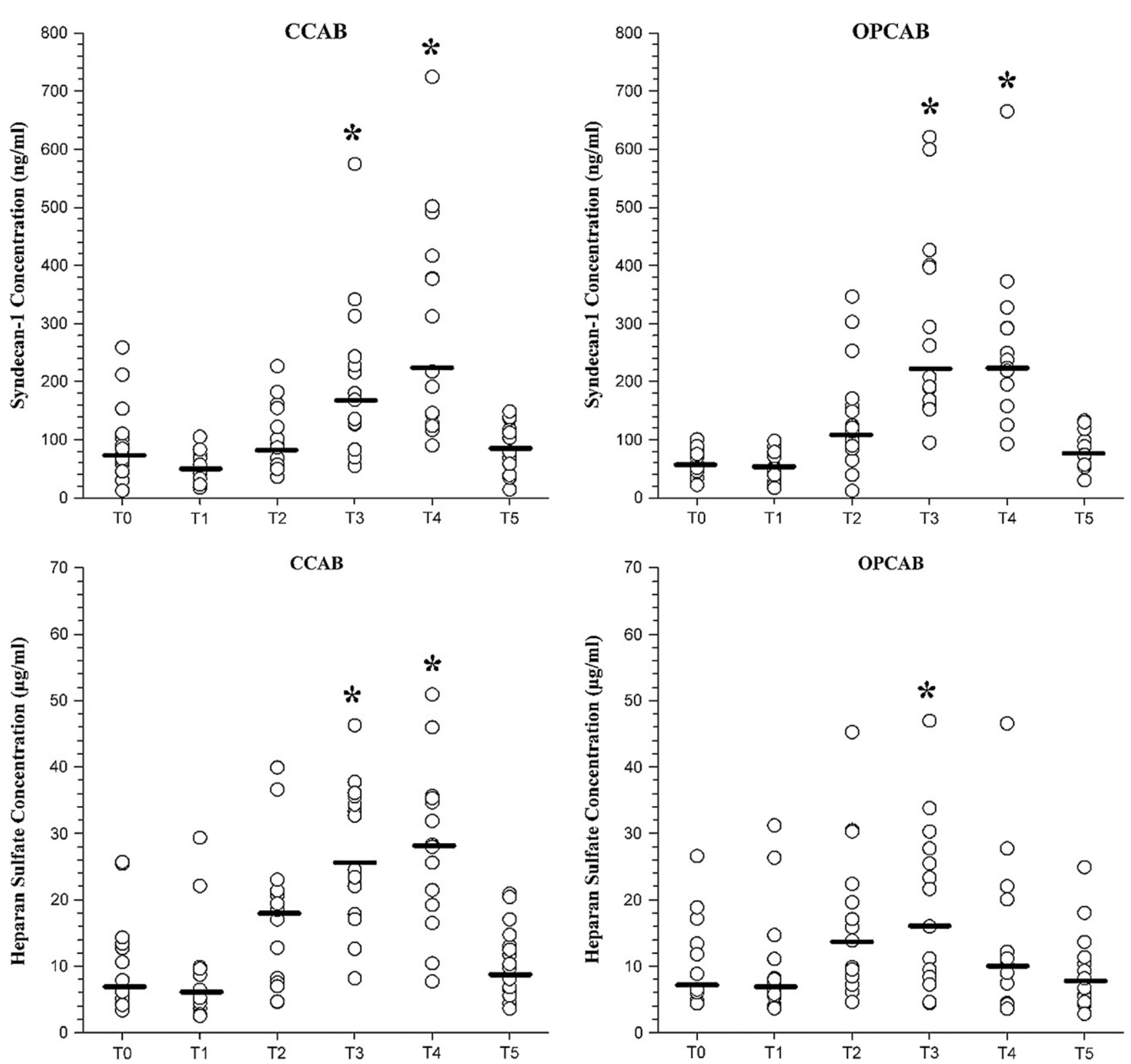

FIGURE 1. Individual and median values of syndecan 1 and heparan sulfate (upper and bottom panels, respectively) in arterial blood plasma of patients undergoing conventional coronary artery bypass grafting $(C C A B$; left panels) and patients undergoing off pump coronary artery bypass grafting $(O P C A B$; right panels) at different operative stages. Time points are as defined in the text. Syndecan 1 was determined directly by using an enzyme linked immuno sorbent assay (Diaclone Research, Besancon, France). Heparan sulfate concentration was measured after pretreatment of plasma with Actinase E (Sigma, St Louis, Mo) with a special enzyme linked immunosorbent assay kit (Seikagaku Corporation, Tokyo, Japan). $* P<.05$ versus T0 (Wilcoxon signed rank test; $P$ value was corrected according to the Bonferroni adjustment for testing 5 times).

patients undergoing coronary artery bypass surgery. Such an origin is suggested by the immunohistochemical localization of both syndecan-1 and heparan sulfate at the luminal side of the endothelium and by former experiments showing postischemic reperfusion to coincide with multifold increases in syndecan-1 and heparan sulfate levels and a visible destruction of the endothelial glycocalyx. ${ }^{3}$ Various animal models showed this structure to be fundamentally involved in nu- merous physiologic and pathophysiologic actions in the circulatory system. Preservation of the glycocalyx should maintain the endothelial permeability barrier and attenuate blood cell vessel wall interactions, thereby mitigating inflammation and tissue edema. ${ }^{1,2}$

Because shedding of the glycocalyx was observed in the present study in both the CCAB and OPCAB groups, ischemia reperfusion injury does not appear to be the sole trigger 

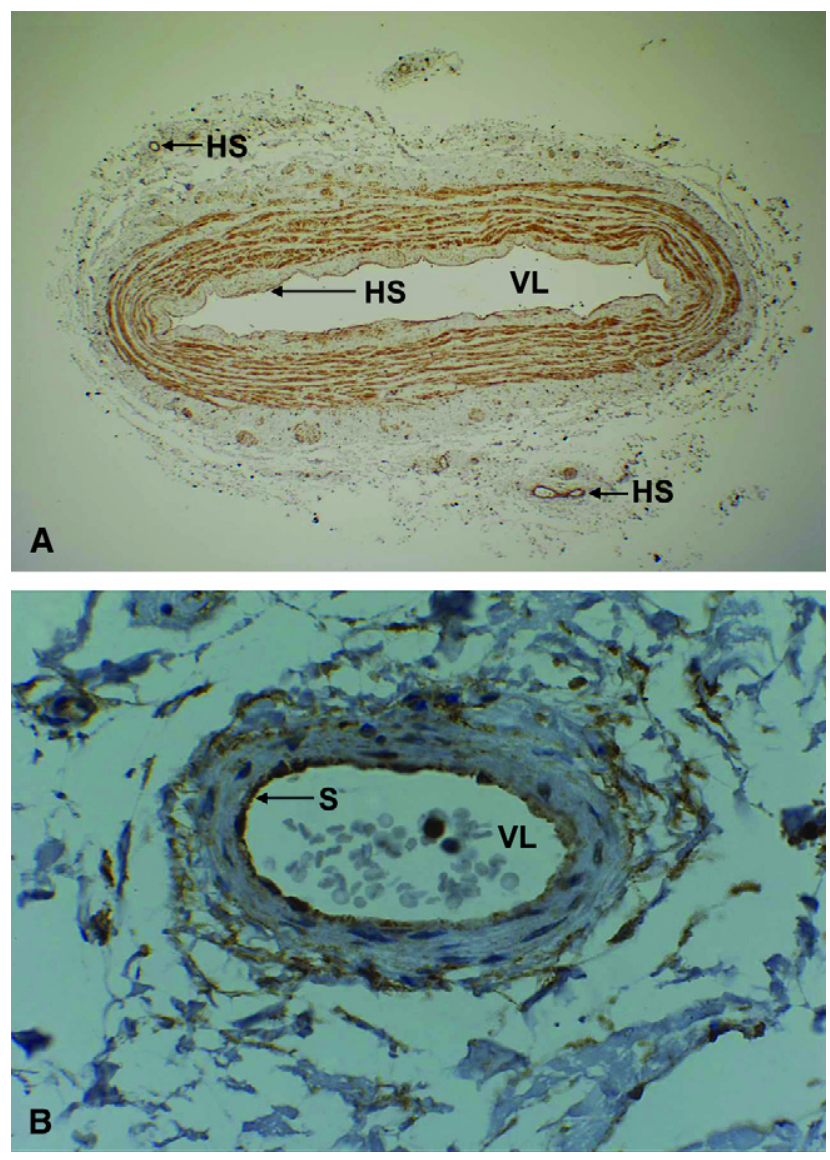

FIGURE 2. Immunostaining of a human saphenous vein and adventitial artery with monoclonal antibody against heparan sulfate (A) and synde can 1 (B). Sections $(5 \mu \mathrm{m})$ were immunohistochemically stained with monoclonal antibodies (heparan sulfate: Seikagaku Corporation, Tokyo, Japan; syndecan 1: Biosource International, Camarillo, Calif). (Original magnification: A, $2 \times$; B, $40 \times . H S$, Heparan sulfate; $V L$, vascular lumen; $S$, syndecan 1 . for disruption. During CCAB and OPCAB surgery, other potential mechanisms, such as protease activation, increased levels of proinflammatory cytokines, or oxidized lipoproteins, might account for destruction of the glycocalyx. ${ }^{2}$ In addition, mechanical stress should cause liberation of atrial natriuretic peptide. Shedding of the endothelial glycocalyx caused by atrial natriuretic peptide was demonstrated in guinea pig hearts. ${ }^{4}$ Given the importance of the glycocalyx, further studies evaluating the mechanisms behind shedding of the human glycocalyx are needed.

\section{References}

1. Pries AR, Secomb TW, Gaehtgens P. The endothelial surface layer. Pflugers Arch. 2000;440:653-66

2. Reitsma S, Slaaf DW, Vink H, van Zandvoort MA, oude Egbrink MG. The endothelial glycocalyx: composition, functions, and visualization. Pflugers Arch. 2007; 454:345-59.

3. Rehm M, Bruegger D, Christ F, Conzen P, Thiel M, Jacob M, et al. Shedding of the endothelial glycocalyx in patients undergoing major vascular surgery with global and regional ischemia. Circulation. 2007;116:1896-906.

4. Bruegger D, Jacob M, Rehm M, Loetsch M, Welsch U, Conzen P, et al. Atrial natriuretic peptide induces shedding of the endothelial glycocalyx in the coronary vascular bed of guinea pig hearts. Am J Physiol Heart Circ Physiol. 2005;289: H1993-9. 